\title{
Impact of pretreatment anemia on upfront abiraterone acetate therapy for metastatic hormone-sensitive prostate cancer: a multicenter retrospective study
}

Teppei Okamoto ${ }^{1}$, Daisuke Noro ${ }^{1}$, Shingo Hatakeyama ${ }^{1 *}$ (D), Shintaro Narita², Koji Mitsuzuka ${ }^{3}$, Toshihiko Sakurai ${ }^{4}$, Sadafumi Kawamura ${ }^{5}$, Senji Hoshi', Jiro Shimoda ${ }^{7}$, Toshikazu Tanaka ${ }^{8}$, Toshiaki Kawaguchi ${ }^{8}$, Shigeto Ishidoya ${ }^{9}$, Akihiro Ito $^{3}$, Norihiko Tsuchiya ${ }^{4}$, Tomonori Habuchi ${ }^{2}$ and Chikara Ohyama ${ }^{1}$

\begin{abstract}
Background: Anemia has been a known prognostic factor in metastatic hormone-sensitive prostate cancer (mHSPC). We therefore examined the effect of anemia on the efficacy of upfront abiraterone acetate (ABI) in patients with mHSPC.

Methods: We retrospectively evaluated $66 \mathrm{mHSPC}$ patients with high tumor burden who received upfront ABI between 2018 and 2020 (upfront ABI group). We divided these patients into two groups: the anemia-ABI group (hemoglobin < $13.0 \mathrm{~g} / \mathrm{dL}, n=20)$ and the non-anemia-ABI group $(n=46)$. The primary objective was to examine the impact of anemia on the progression-free survival (PFS; clinical progression or PC death before development of castration resistant PC) of patients in the upfront $\mathrm{ABI}$ group. Secondary objectives included an evaluation of the prognostic significance of upfront $\mathrm{ABI}$ and a comparison with a historical cohort (131 mHSPC patients with high tumor burden who received androgen deprivation therapy (ADT/complete androgen blockade [CAB] group) between 2014 and 2019).

Results: We found that the anemia-ABI group had a significantly shorter PFS than the non-anemia-ABI group. A multivariate Cox regression analysis showed that anemia was an independent prognostic factor of PFS in the upfront $\mathrm{ABI}$ group (hazard ratio, 4.66; $P=0.014$ ). Patients in the non-anemia-ABI group were determined to have a significantly longer PFS than those in the non-anemia-ADT/CAB group $(n=68)(P<0.001)$. However, no significant difference was observed in the PFS between patients in the anemia-ABI and the anemia-ADT/CAB groups $(n=63)$. Multivariate analyses showed that upfront $\mathrm{ABI}$ could significantly prolong the PFS of patients without anemia (hazard ratio, $0.17 ; P<0.001$ ), whereas $A B I$ did not prolong the PFS of patients with anemia.
\end{abstract}

Conclusion: Pretreatment anemia was a prognostic factor among mHSPC patients who received upfront ABI. Although the upfront ABI significantly improved the PFS of mHSPC patients without anemia, its efficacy in patients with anemia might be limited.

Keywords: Prostate cancer, Hormone-sensitive, Abiraterone acetate, Upfront therapy, Prognosis, Anemia

\footnotetext{
* Correspondence: shingoh@hirosaki-u.ac.jp; shingorilla2@gmail.com

'Department of Urology, Department of Advanced Blood Purification

Therapy, Hirosaki University Graduate School of Medicine, 5 Zaifu-chou, Hirosaki 036-8562, Japan

Full list of author information is available at the end of the article
}

\section{$\triangle B M C$}

(c) The Author(s). 2021 Open Access This article is licensed under a Creative Commons Attribution 4.0 International License, which permits use, sharing, adaptation, distribution and reproduction in any medium or format, as long as you give appropriate credit to the original author(s) and the source, provide a link to the Creative Commons licence, and indicate if changes were made. The images or other third party material in this article are included in the article's Creative Commons. licence, unless indicated otherwise in a credit line to the material. If material is not included in the article's Creative Commons licence and your intended use is not permitted by statutory regulation or exceeds the permitted use, you will need to obtain permission directly from the copyright holder. To view a copy of this licence, visit http://creativecommons.org/licenses/by/4.0/ The Creative Commons Public Domain Dedication waiver (http://creativecommons.org/publicdomain/zero/1.0/) applies to the data made available in this article, unless otherwise stated in a credit line to the data. 


\section{Background}

Prostate cancer (PC) is one of the most prevalent cancers in men worldwide $[1,2]$. In Japan, approximately $10 \%$ of patients with PC initially present with distant metastases [3]. Most of these patients experience eventually progression to metastatic castration-resistant PC (CRPC) despite good initial response to androgen deprivation therapy (ADT) $[4,5]$. Recently, the LATI TUDE trial demonstrated that upfront abiraterone acetate (ABI, a type of androgen receptor-targeted agent [ARTA]) added to ADT can lead to significant benefits in mHSPC patients compared with ADT monotherapy $[6,7]$. However, these studies showed an almost similar PFS and overall survival between the ADT monotherapy and upfront ABI groups in the early term of the study $[6,7]$. Thus, we speculated that some important prognostic and/or predictive factors may be present among patients who received ABI therapy.

Anemia is a powerful prognostic factor in PC $[5,8]$. Our previous study demonstrated that pretreatment anemia was an independent prognostic factor that predicted oncological outcomes among mHSPC patients treated with ADT monotherapy or complete androgen blockade (CAB) [5]. However, the prognostic significance of anemia among mHSPC patients treated with ARTA remains unclear. Therefore, we retrospectively examined the prognostic significance of pretreatment anemia on the oncological outcomes of mHSPC patients treated with upfront ABI.

\section{Methods}

This retrospective study was performed according to the ethical standards of the Declaration of Helsinki and was further approved by the ethics review board of the Hirosaki University School of Medicine (authorization number: 2019-094).

\section{Study population and patient selection}

In total, 168 mHSPC patients with high tumor burden, who were initially treated with $\mathrm{ADT}$ alone or $\mathrm{CAB}$ (ADT/CAB, $n=101)$ or upfront ABI therapy $(n=67)$, were retrospectively examined at the Hirosaki University Hospital and associated hospitals between 2008 and 2020 (Aomori database). Furthermore, we retrospectively evaluated 563 mHSPC patients with CHAARTED highvolume disease, who were in the Michinoku Japan Urological Cancer Study Group database and who were initially treated with ADT/CAB between 2008 and 2016 (Michinoku database) $[4,5,9]$. Considering that patients with metastatic CRPC had a chance to receive ARTAs and improved their prognosis, the era of diagnosis or treatment might be associated with prognoses among mHSPC patients. We therefore excluded 22 patients treated with ADT/CAB who were diagnosed before 2014 in the Aomori database and 131 in the Michinoku database. We excluded 22 patients treated with $A D T / C A B$ in the Aomori database and 354 in the Michinoku database due to insufficient baseline laboratory data, such as information on hemoglobin $(\mathrm{Hb})$, lactate dehydrogenase (LDH), and alkaline phosphatase (ALP) (Fig. S1). In addition, we excluded 5 patients ( 1 for upfront $A B I$ group and 4 for $\mathrm{ADT} / \mathrm{CAB}$ group) who died of other than PC before the development of CRPC. Finally, 197 mHSPC patients who had high tumor burden were evaluated (Fig. 1); they were then divided into two groups: the ADT/CAB group $(n=131)$ and the upfront $\mathrm{ABI}$ group $(n=66)$. We further categorized the patients into the following groups based on whether they had pretreatment anemia: non-anemia-ABI $(n=46)$, anemiaABI $(n=20), \quad$ non-anemia-ADT/CAB $(n=68), \quad$ and anemia-ADT/CAB groups $(n=63)$.

\section{Variable evaluations}

The following variables were examined at diagnosis: age, Eastern Cooperative Oncology Group Performance Status (ECOG-PS), Gleason score, and initial prostatespecific antigen (PSA), $\mathrm{Hb}, \mathrm{LDH}$, and ALP levels. ECOG-PS was defined as worse if ECOG-PS was of $\geq 1$ [10]. The cutoff value of PSA level was set at $100 \mathrm{ng} / \mathrm{mL}$ [11]. Anemia was defined as an $\mathrm{Hb}$ level $<13.0 \mathrm{~g} / \mathrm{dL}$ [12]. The cutoff values for LDH and ALP levels were set at 222 and $322 \mathrm{IU} / \mathrm{L}$, respectively, based on the standard common reference for clinical laboratory tests in Japan [13]. We evaluated metastatic status before treatment using chest and body computed tomography and bone scintigraphy scans. Bone metastatic volume was assessed according to the extent of disease (EOD) score. EOD scores were divided into 5 based on bone scintigraphy as follows [14]. Score 0, normal or abnormal due to benign bone lesion; Score 1, less than 6 metastatic bone lesions, each of which is less than $50 \%$ the size of a vertebral body; Score 2, the number of metastatic bone lesions is between 6 and 20; Score 3, the number of metastatic bone lesions is more than 20 but less than a "super scan"; and Score 4, "super scan". CRPC-free survival was evaluated from the date of the initial diagnosis of mHSPC to the date of CRPC diagnosis according to the recommendations of the Cancer Clinical Trials Working Group 2 [15].

\section{Treatment protocol}

Patients were initially treated with ADT alone or ADT plus bicalutamide before upfront ABI therapy was approved. Starting in March 2018, patients who met the LATITUDE study criteria could receive upfront $A B I$ therapy. 


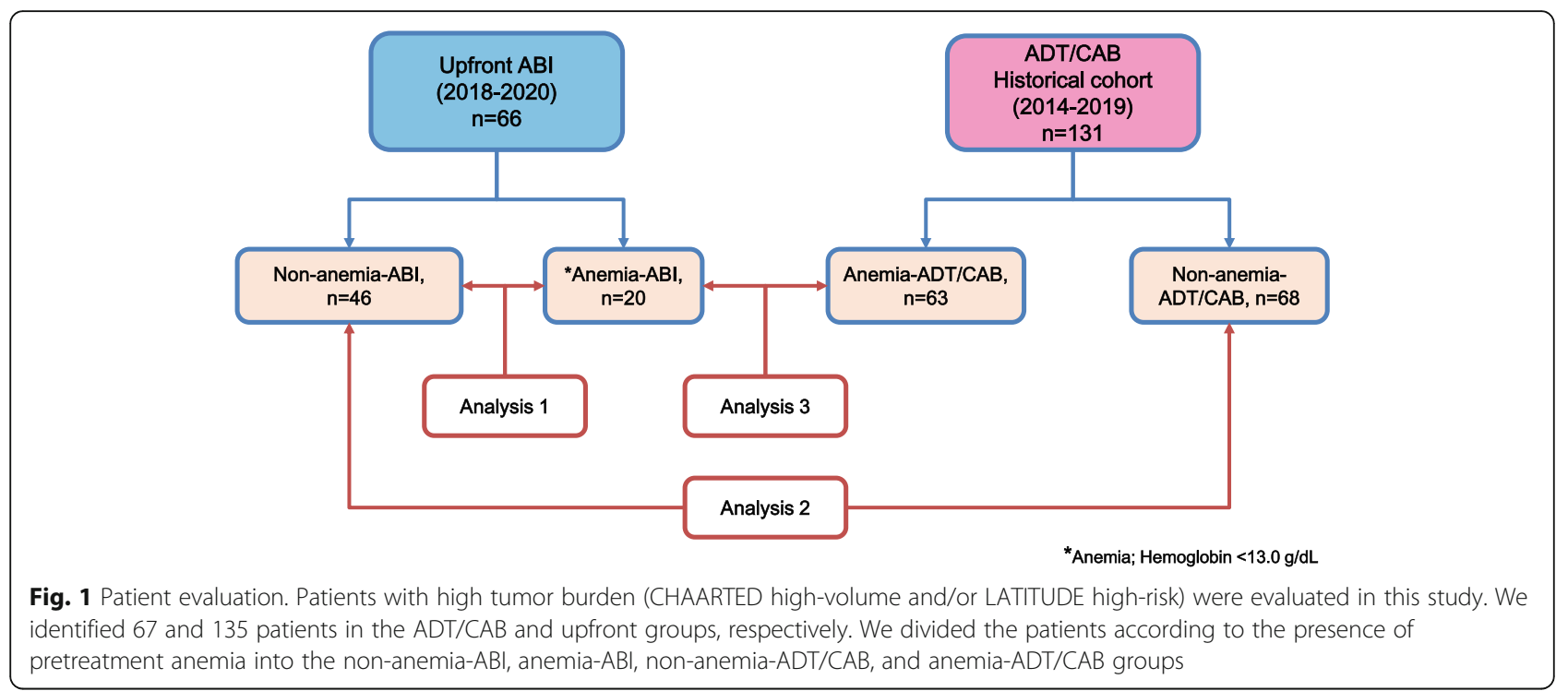

\section{Primary objective}

Our primary purpose was to evaluate cancer progression-free survival (PFS) (i.e., development of CRPC or PC death before the development of CRPC). We compared the clinical PFS between patients in the anemia-ABI and those in the nonanemia-ABI groups (Analysis-1). The adverse event (AE)-related discontinuation of ABI was compared between patients with and without anemia. We then assessed the impact of anemia and other clinical factors on the PFS of patients in the upfront ABI group using Cox proportional hazard analyses. We adopted the propensity score-based inverse probability of treatment weighting (IPTW) method in order to adjust for group imbalances.

\section{Secondary objectives}

The secondary objectives of this study were to compare the PFS between patients in the non-anemia-ABI group and those in the non-anemia-ADT/CAB group (Analysis-2), and between patients in the anemia-ABI group and those in the anemia-ADT/CAB group (Analysis-3). We then assessed the efficacy of upfront ABI on the PFS using multivariate Cox proportional hazard analyses or IPTW-adjusted analyses.

\section{Statistical analysis}

Statistical analyses were conducted using GraphPad Prism 5.03 (GraphPad Software, San Diego, CA, USA), EzR (R commander version 1.6-3), and R 3.3.2 (The R Foundation for Statistical Computing, Vienna, Austria). Categorical variables were compared using Fisher's exact test or chi-squared test. Quantitative variables are then expressed as means with standard deviations or as medians with interquartile ranges (IQRs). The statistical difference between groups was compared using Student's $t$-test for normally distributed data or Mann-Whitney $U$-test for non-normally distributed data. The PFS was estimated and compared using the Kaplan-Meier curve and log-rank test, respectively. In case of limited event numbers, we adopted an IPTW-adjusted multivariable Cox regression analysis. Hazard ratios (HRs) with $95 \%$ confidence intervals (CIs) were calculated after controlling for potential confounders of PC, such as age, ECOGPS, initial PSA, Gleason score, visceral metastasis, EOD, and ALP and LDH levels. We also conducted a simultaneous multivariate Cox regression analysis of PFS. $P$-values $<0.05$ were considered to be statistically significant.

\section{Results}

\section{Primary objective}

The patients in the anemia-ABI had significantly a worse ECOG-PS $(P=0.039)$, higher initial PSA $(P=0.001)$, EOD $(P=0.002)$, ALP $(P=0.010)$, and LDH $(P=0.017)$ than those in the non-anemia-ABI group (Table 1 ). Three patients discontinued ABI therapy due to severe AEs (two for liver damage and one for hyponatremia). No difference was observed in the rate of AE-related discontinuation of ABI between the two groups. During the median follow-up period of 13 months, 15 patients in the upfront ABI group experienced clinical progression (Table 1). Fourteen patients developed CRPC (nine in the anemia-ABI group), and two patients in the anemia-ABI group died before the development of CRPC. Of the 14 patients who developed CRPC, 2 died from PC. We observed a 
Table 1 Upfront ABI group

\begin{tabular}{|c|c|c|c|}
\hline & Anemia & Non-anemia & $P$ value \\
\hline$n$ & 20 & 46 & \\
\hline $\mathrm{Age}^{\mathrm{a}}$ (years) (mean, SD) & $72(6)$ & $71(6)$ & 0.231 \\
\hline ECOG-PS ${ }^{b}$ (median, IQR) & $0(0-1)$ & $0(0-0)$ & 0.039 \\
\hline $\begin{array}{l}\text { Initial PSA }{ }^{\mathrm{b}}(\mathrm{ng} / \mathrm{mL}) \\
\text { (median, IQR) }\end{array}$ & 961 (314-2702) & $189(51-448)$ & 0.001 \\
\hline $\begin{array}{l}\text { Gleason score } \\
\text { (median, IQR) }\end{array}$ & $9(8-10)$ & $9(8-9)$ & 0.649 \\
\hline Visceral metastasis $^{c}$ & $3(15 \%)$ & $11(24 \%)$ & 0.524 \\
\hline Lung & 2 & 11 & - \\
\hline Liver & 1 & 0 & - \\
\hline Others & 0 & 0 & - \\
\hline $\mathrm{EOD}^{\mathrm{b}}$ (median, IQR) & $3(2-3)$ & $2(2-3)$ & 0.002 \\
\hline ALP ${ }^{\mathrm{b}}(\mathrm{IU} / \mathrm{L})$ (median, IQR) & 949 (372-1684) & $357(258-717)$ & 0.010 \\
\hline $\mathrm{LDH}^{\mathrm{b}}$ (IU/L) (median, IQR) & $233(200-279)$ & $199(166-227)$ & 0.017 \\
\hline $\begin{array}{l}\text { Discontinuation of } A B I \\
\text { due to } A E s^{c}\end{array}$ & $1(5.0 \%)$ & $2(4.3 \%)$ & 1.000 \\
\hline $\begin{array}{l}\text { Clinical progression }{ }^{c} \\
\text { (CRPC or PC death } \\
\text { before CRPC) }\end{array}$ & $10(50 \%)$ & $5(11 \%)$ & $<0.001$ \\
\hline $\mathrm{CRPC}^{\mathrm{C}}$ & $9(43 \%)$ & $5(11 \%)$ & 0.002 \\
\hline PC death before CRPC $^{C}$ & $1(5.0 \%)$ & $0(0 \%)$ & 0.303 \\
\hline Death after CRPC $^{\mathrm{C}}$ & $1(5.0 \%)$ & $1(2.3 \%)$ & 0.518 \\
\hline $\begin{array}{l}\text { Follow-up period }{ }^{\mathrm{b}} \text { (months) } \\
\text { (median, IQR) }\end{array}$ & $11(11-15)$ & $13(11-17)$ & 0.130 \\
\hline
\end{tabular}

$A B I$ abiraterone acetate, $S D$ standard deviation, ECOG-PS Eastern Cooperative Oncology Group performance status, IQR interquartile range, $P S A$ prostate specific antigen, $E O D$ extent of disease, $A L P$ alkaline phosphatase, $L D H$ lactate dehydrogenase, $A E$ adverse event, $P C$ prostate cancer, $C R P C$ castration resistant prostate cancer

${ }^{a}$ Student $t$ test

${ }^{\mathrm{b}}$ Mann-Whitney $\mathrm{U}$ test

' $\mathrm{X} 2$ test or Fisher's exact test

significantly longer PFS in the non-anemia-ABI group compared with that of the anemia-ABI group $(P<0.001)$ (Fig. 2a). Univariate Cox regression analyses in the ABI group showed that an LDH level $\geq 222 \mathrm{IU} / \mathrm{L}$ (HR, 3.70; $P=0.019)$ and anemia $(\mathrm{HR}, 5.58 ; P=0.002)$ were significantly associated with worse PFS (Table 2). IPTW-adjusted multivariate Cox regression analyses revealed that anemia ( $\mathrm{HR}, 4.66 ; P=0.014)$ was independently associated with worse PFS, while an LDH level $\geq 222 \mathrm{IU} / \mathrm{L}$ was not (Fig. 2b). A simultaneous multivariate Cox regression analysis of PFS showed that only anemia was an independent factor (HR, 5.64; $P=0.016$; Table 3).

\section{Secondary objectives}

Table S2 illustrates a comparison of the characteristics between patients in the upfront ABI and ADT/
$\mathrm{CAB}$ groups, according to the presence or absence of anemia. No significant group-difference was observed other than follow-up period between the non-anemia$A B I$ and non-anemia-ADT/CAB group, between the anemia-ABI and anemia-ADT/CAB group. Our additional study showed that patients in the upfront $A B I$ group had a significantly longer PFS than those in the $\mathrm{ADT} / \mathrm{CAB}$ group in the entire cohort $(P<0.001)$ (Fig. S2A). Similarly, the PFS in the non-anemia-ABI group was significantly superior to that in the nonanemia-ADT/CAB group $(P<0.001)$ (Fig. 3a). However, the PFS of patients in the anemia-ABI group did not have a significantly longer than that of patients in the anemia-ADT/CAB group $(P=0.358)$ (Fig. 3b). The IPTW adjusted-multivariable Cox regression analysis revealed that upfront ABI therapy was significantly associated with prolonged PFS in patients without anemia (HR, 0.17; $P<0.001$ ) (Fig. 3c top), whereas this was not observed in patients with anemia (HR, 0.54; $P=0.186$ ) (Fig. 3c bottom).

\section{Discussion}

We compared the PFS between mHSPC patients with anemia and those without anemia in the upfront ABI group. We found an independent association between pretreatment anemia and the efficacy of upfront ABI therapy. As the LATITUDE trial has shown [6, 7], our study demonstrated the efficacy of upfront ABI therapy for mHSPC patients with hightumor burden. This trend was remarkable especially in patients without anemia. However, we did not observe any meaningful difference in PFS between patients with anemia in the upfront $A B I$ group and those in the ADT/CAB group. To the best of our knowledge, this is the first study demonstrating between pretreatment anemia and clinical outcome in patients treated with ARTA.

Anemia has been identified as one of the most prevalent characteristics of malignancies [16]. Among mHSPC patients, the prevalence of pretreatment anemia was as high as $44-50 \%[5,17]$. This study showed that the prevalence of anemia in this entire cohort was $43 \%$. Anemia in mHSPC patients may be attributed to several factors, including malnutrition and chronic inflammation [5, 18]. Our previous study demonstrated that $85 \%$ of $\mathrm{mHSPC}$ patients with malnutrition were diagnosed with anemia [5]. Furthermore, the $\mathrm{Hb}$ level was inversely correlated with interleukin- 6 expression, oxidative stress markers, and C-reactive protein level among patients with advanced cancer, which implied that inflammatory status is associated with worse iron metabolism in advanced cancers [16]. The relationship among anemia, malnutrition, and inflammatory status may be largely 


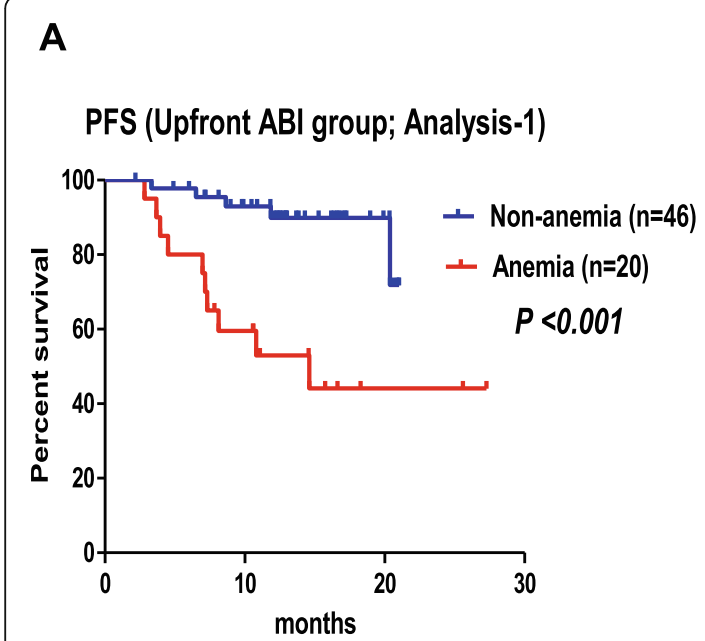

B IPTW adjusted-multivariate Cox regression analyses for PFS
in the upfront $A B I$ group

Fig. 2 Comparison of clinical progression (Analysis-1). a PFS between patients in the anemia-ABI and non-anemia-ABI groups (Analysis-1) (median, not reached vs. 15 months; $P<0.001$ ). $\mathbf{b}$ IPTW adjusted-multivariate logistic regression analyses for PFS in the upfront ABI group (adjustments for age, ECOG-PS, initial PSA, Gleason score, visceral metastasis, EOD, and ALP and LDH/Hb levels). Anemia (HR, 4.66; 95\% Cl, 1.36$16.0 P=0.014)$. LDH level > $222 \mathrm{IU} / \mathrm{L}(\mathrm{HR}, 2.68 ; 95 \% \mathrm{Cl}, 0.81-8.82 P=0.105)$

explained by cancer cachexia. Cancer cachexia is a complex syndrome that is often characterized by severe fatigue and progressive weight loss and is caused by a cancer-induced negative protein and energy balance as well as by inflammation. Malnutrition and inflammation suppress protein synthesis, particularly in advance caners, and it may also suppress erythropoiesis [19]. Another possible explanation for anemia in mHSPC patients is bone marrow infiltration. In this study, patients in the anemia-ABI group had a significantly worse EOD than those in the non-anemia-ABI group. Additionally, our study demonstrated that EOD was an independent factor of pretreatment

Table 2 Univariate Cox hazard proportional analyses for progression-free survival in the upfront $A B I$ group

\begin{tabular}{lllll}
\hline Variables & Factor & $\boldsymbol{P}$ value & Hazard ratio & $\mathbf{9 5 \%} \mathbf{~ C l}$ \\
\hline Age & $\geq 75$ years & 0.780 & 0.85 & $0.27-2.78$ \\
ECOG-PS & $\geq 1$ & 0.150 & 2.09 & $0.76-5.75$ \\
Initial PSA & $\geq 100 \mathrm{ng} / \mathrm{ml}$ & 0.230 & 2.60 & $0.54-12.5$ \\
Gleason score & $\geq 9$ & 0.438 & 0.67 & $0.24-1.85$ \\
Visceral metastasis & presence & 0.380 & 0.51 & $0.11-2.29$ \\
EOD & $0-4$ & 0.375 & 1.29 & $0.74-2.24$ \\
ALP & $\geq 322 \mathrm{IU} / \mathrm{L}$ & 0.290 & 1.97 & $0.56-6.98$ \\
LDH & $\geq 222 \mathrm{IU} / \mathrm{L}$ & 0.019 & 3.70 & $1.24-11.0$ \\
Anemia & presence & 0.002 & 5.58 & $1.90-16.4$ \\
\hline
\end{tabular}

$A B I$ abiraterone acetate, $C l$ confidence interval, ECOG-PS Eastern Cooperative Oncology Group performance status, PSA prostate specific antigen, EOD extent of disease, $A L P$ alkaline phosphatase, $L D H$ lactate dehydrogenase anemia (Odds ratio, 1.93) in the entire cohort (Fig. $\mathrm{S} 2 \mathrm{~B})$. Anemia due to replacement of normal bone marrow with cancer cells is termed leucoerythroblastic anemia [18], which is observed in approximately $30 \%$ of patients with metastatic CRPC [20]. These findings suggest that anemia among advanced PC patients may reflect cancer cachexia and/or disease aggressiveness.

The relationship between anemia and prognosis in patients with metastatic PC is debatable. A metaanalysis demonstrated that of the mHSPC patients in the ADT/CAB group, those with anemia had a significantly shorter PFS than those without anemia [8]. Tumor hypoxia caused by anemia and ADT may explain the relationship between anemia and poor prognosis in metastatic PC. Cancer-associated systemic anemia can cause decreased oxygen transport capacity of the blood, which contributes to hypoxia in the tumor microenvironment $[21,22]$. Hypoxia may decrease tumor control through the induction of hypoxia-inducible factor $1 \alpha$ (HIF-1 $\alpha$ ) [23]. HIF-1 $\alpha$ has been identified to activate HIF-1 $\beta$ to form a transcription factor complex that regulates the expression of several genes, such as vascular endothelial growth factor and glucose transporters, which is strongly associated with angiogenesis and tumor growth [24, 25]. Tumor hypoxia is often associated with treatment resistance in other cancers [23]. Tumor hypoxia in PC cell was associated with clinical stage and biochemical recurrence in locally advanced prostate cancer [26, 27]. Furthermore, ADT may accelerate hypoxia in PC 
Table 3 Simultaneous multivariate Cox regression analysis for PFS in the upfront ABI group

\begin{tabular}{lllll}
\hline Variable & Factor & $\boldsymbol{P}$ value & Hazard ratio & $\mathbf{9 5 \%} \mathbf{C l}$ \\
\hline Age $\geq 75$ years & Presence & 0.280 & 0.51 & $0.15-1.73$ \\
ECOG-PS $\geq 1$ & Presence & 0.750 & 1.15 & $0.35-3.81$ \\
Initial PSA $\geq 100 \mathrm{ng} / \mathrm{ml}$ & Presence & 0.810 & 0.92 & $0.14-5.96$ \\
Gleason score $\geq 9$ & Presence & 0.910 & 0.94 & $0.31-2.82$ \\
Visceral metastasis & Presence & 0.760 & 0.67 & $0.13-3.46$ \\
EOD & $0-4$ & 0.380 & 0.74 & $0.38-1.44$ \\
ALP $\geq 322$ IU/L & Presence & 0.940 & 0.94 & $0.19-4.59$ \\
LDH $\geq 222$ IU/L & Presence & 0.055 & 3.51 & $0.97-12.3$ \\
Anemia & Presence & 0.016 & 5.64 & $1.39-20.9$ \\
\hline
\end{tabular}

PFS progression-free survival, $A B I$ abiraterone acetate, $\mathrm{Cl}$ confidence interval, ECOG-PS Eastern Cooperative Oncology Group performance status, PSA prostate specific antigen, EOD extent of disease, ALP alkaline phosphatase, $L D H$ lactate dehydrogenase

cells, which ultimately results in CRPC [28]. Although the relationship between tumor hypoxia and responsiveness to ADT in mHSPC remains unclear, we speculated that poor clinical outcomes of mHSPC patients with anemia might be related with tumor hypoxia. Therefore, improvement in the prognosis of mHSPC patients with anemia is a key issue for clinicians.

Optimal selection of upfront intensive therapies for mHSPC patients with anemia must be carefully considered. Patients with anemia and/or malnutrition (cancer cachexia) are believed to be intolerant to chemotherapies [29]. In this regard, patients with anemia and/or malnutrition should be treated with upfront $A B I$ therapy rather than upfront docetaxel. Indeed, the rate of AE-related discontinuation of $\mathrm{ABI}$ among patients with anemia was not very high in this study. However, our results implied that the efficacy of upfront ABI therapy might be limited in mHSPC patients with anemia. As no clinical trial of ARTAs focused on the impact of anemia on prognosis, further studies are necessary to confirm our findings. Careful selection for upfront $\mathrm{ABI}$ therapy might be necessary in mHSPC patients with anemia.

This study included a small sample size and a short-term follow-up period, which precluded a definitive conclusion regarding the long-term survival benefit. We did not obtain a full dataset on nutritional status and other immeasurable confounding factors. In this study, we therefore had to exclude 376 patients who received $\mathrm{ADT} / \mathrm{CAB}$ due to lacking essential data, which resulted in major selection bias. We did not have data on baseline serum testosterone levels which is one of important factor of prostate cancer and anemia. Our results may not be applicable to other countries because of racial and regional differences. Despite these limitations, we revealed the prognostic significance of pretreatment anemia among mHSPC patients who received upfront $\mathrm{ABI}$ therapy.

\section{Conclusion}

The upfront ABI therapy significantly improved the PFS of mHSPC patients, especially those without anemia. However, its efficacy in patients with anemia

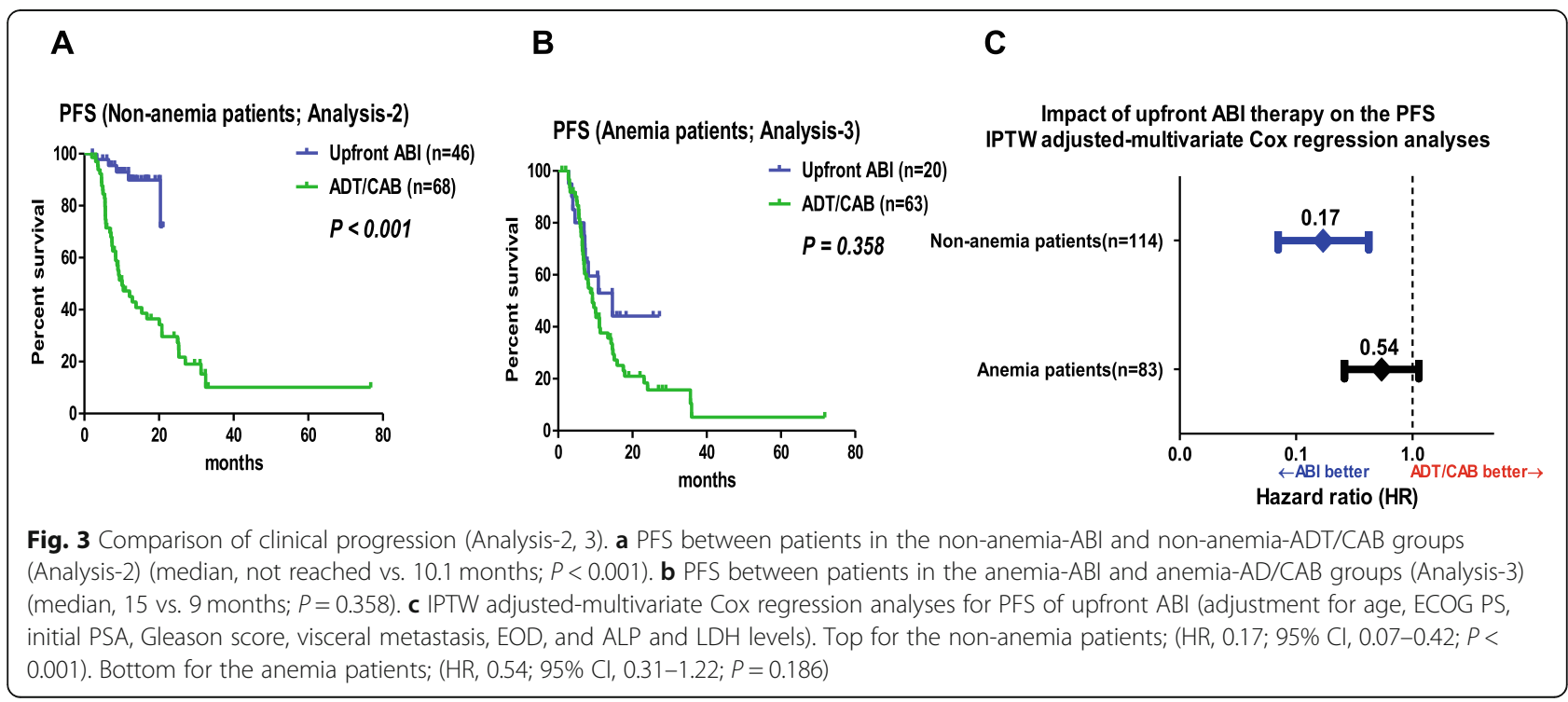


might be limited. Studies with a larger sample size and longer follow-up are needed to confirm our results.

\section{Abbreviations}

mHSPC: metastatic hormone-sensitive prostate cancer; ABI: Abiraterone acetate; PFS: Progression-free survival; ARTA: Androgen receptor-targeted agent; ADT: Androgen deprivation therapy; CAB: Complete androgen blockage; PC: Prostate cancer; CRPC: Castration resistant prostate cancer; Hb: Hemoglobin; LDH: Lactate dehydrogenase; ALP: Alkaline phosphatase; ECOG-PS: Eastern Cooperative Oncology Group Performance Status; PSA: Prostate specific antigen; EOD: Extent of disease; AE: Adverse event; IPTW: Inverse probability of treatment weighting; HR: Hazard ratio; Cl: Confidence interval; HIF: Hypoxia-inducible factor

\section{Supplementary Information}

The online version contains supplementary material available at https://doi. org/10.1186/s12885-021-08206-8

\section{Additional file 1: Figure S1. Patient selection. We retrospectively} evaluated $168 \mathrm{mHSPC}$ patients with high tumor burden who were initially treated with ADT alone or CAB (ADT/CAB, $n=101)$ or upfront $A B$ therapy $(n=67)$ in the Aomori database. Furthermore, we retrospectively evaluated $563 \mathrm{mHSPC}$ patients with high-volume disease, as reported in the CHAARTED trial, in the Michinoku database. We excluded 44 patients who were treated with ADT/CAB in the Aomori database and 485 patients in the Michinoku database due to old era of initial diagnosis (22 patients in the Aomori database and 131 in the Michinoku database) or insufficient baseline laboratory data or (22 patients in the Aomori database and 354 in the Michinoku database). Figure S2 Comparison of clinical progression in the entire cohort and multivariate logistic analysis for pretreatment anemia. (A) PFS between patients in the upfront $A B I$ and ADT/CAB groups and those in the entire cohort (median, not reached vs. 10 months; $P<0.001$ ). (B) EOD (odds ratio [OR], 1.93; 95\% Cl, 1.27-1.93; $P=0.002)$ and age $\geq 75$ years $(O R, 3.16 ; 95 \% C l, 1.64-6.10 ; P<0.001)$ were independent factors for pretreatment anemia. The model was adjusted by $A L P \geq 322 \mathrm{IU} / \mathrm{L}, \mathrm{LDH} \geq 222 \mathrm{IU} / \mathrm{L}$, ECOG-PS $\geq 1$, visceral metastasis, Gleason score $\geq 9$, and initial PSA $\geq 100 \mathrm{ng} / \mathrm{ml}$. Variance of inflation factor values of age $\geq 75$ years, ALP $\geq 322 \mathrm{IU} / \mathrm{L}, \mathrm{EOD}, \mathrm{LDH} \geq 222 \mathrm{IU} / \mathrm{L}$, ECOG-PS $\geq 1$, visceral metastasis, Gleason score $\geq 9$, and initial PSA $\geq 100 \mathrm{ng} / \mathrm{ml}$ were $1.07,1.15 .1 .30,1.05,1.12,1.17,1.06$, and 1.24 , respectively, which means no multi-collinearity among covariates were observed.

\section{Acknowledgments}

The authors would like to thank Itsuto Hamano, Yuta Kojima, Yuka Kubota, Masaki Momota, Teppei Matsumoto, Masaaki Oikawa, Naoki Fujita, Hiromichi Iwamura, Yuichiro Suzuki, Naoki Sugiyama, Satoshi Narita, Shoji Nishimura, Kazuaki Yoshikawa, Shinya Takahashi, Toshiaki Kawaguchi, Hiroyuki Ito, Tadafumi Saito, Atsushi Sasaki, Hirotake Kodama, Sakae Konishi, Shogo Hosogoe, Takuma Narita, Noriko Tokui, Hayato Yamamoto, Takahiro Yoneyama, Yasuhiro Hashimoto, and Hisao Saitoh for their invaluable support. Moreover, the authors would like to thank Enago (www.enago.jp) for English language review.

\section{Authors' contributions}

(1) Conception and design: SH1, SN; (2) Acquisition of data: All authors; (3) Data analysis and interpretation: TO, SH1, CO; (4) Manuscript writing: TO, SH1; (5) Final approval of manuscript: All authors.

\section{Funding}

This work was supported by the Japan Society for the Promotion of Science (JSPS) KAKENHI Grant Numbers 18 K16718 (Daisuke Noro), $19 H 05556$ (Chikara Ohyama), and 20 K09517 (Shingo Hatakeyama).

The funder did not have no role in the conception of study design, data collection and analysis, or preparation of the manuscript.

\section{Availability of data and materials}

The datasets during and/or analyzed during the current study available from the corresponding author on reasonable request

\section{Declarations}

\section{Ethics approval and consent to participate}

This retrospective study was performed according to the ethical standards of the Declaration of Helsinki and was further approved by the ethics review board of the Hirosaki University School of Medicine (authorization number: 2019-094). Pursuant to the provisions of the ethics committee and the ethic guideline in Japan, written consent was not required in exchange for public disclosure of study information in the case of retrospective and/or observational study using a material such as the existing documentation.

\section{Consent for publication}

Not applicable.

\section{Competing interests}

All authors have no conflicts of interest to declare.

\section{Author details}

'Department of Urology, Department of Advanced Blood Purification Therapy, Hirosaki University Graduate School of Medicine, 5 Zaifu-chou, Hirosaki 036-8562, Japan. ${ }^{2}$ Department of Urology, Akita University School of Medicine, 1-1-1, Hondo, Akita 010-8543, Japan. '3epartment of Urology, Tohoku University School of Medicine, 2-1 Seiryo-machi, Aoba-ku, Sendai, Miyagi 980-8575, Japan. ${ }^{4}$ Department of Urology, Yamagata University School of Medicine, 2-2-2 lida-Nishi, Yamagata, Yamagata 990-9585, Japan. ${ }^{5}$ Department of Urology, Miyagi Cancer Center, 47-1, Nodayama, Shiote, Aijima, Natori, Miyagi 981-1293, Japan. ${ }^{6}$ Department of Urology, Yamagata Prefectural Central Hospital, 1800, Aoyanagi, Yamagata 990-2292, Japan. ${ }^{7}$ Department of Urology, Iwate Prefectural Isawa Hospital, 61, Ryugabaab, Mizusawa-ku, Oshu, Iwate 023-0864, Japan. ${ }^{8}$ Department of Urology, Aomori Prefectural Central Hospital, 2-1-1, Higashi-tsukurimichi, Aomori, Aomori 030-8553, Japan. ${ }^{9}$ Department of Urology, Sendai City Hospital, 1-1-1, Nagamachi, Asuto, Taihaku-ku, Sendai, Miyagi 982-8502, Japan.

Received: 8 October 2020 Accepted: 16 April 2021

Published online: 25 May 2021

\section{References}

1. Siegel RL, Miller KD, Jemal A. Cancer statistics, 2015. CA Cancer J Clin. 2015; 65(1):5-29. https://doi.org/10.3322/caac.21254.

2. Kimura T, Egawa S. Epidemiology of prostate cancer in Asian countries. Int J Urol. 2018;25(6):524-31. https://doi.org/10.1111/iju.13593.

3. Fujimoto H, Nakanishi H, Miki T, Kubota Y, Takahashi S, Suzuki K, et al. Oncological outcomes of the prostate cancer patients registered in 2004: report from the Cancer registration committee of the JUA. Int J Urol. 2011; 18(12):876-81. https://doi.org/10.1111/j.1442-2042.2011.02895.x.

4. Okita K, Hatakeyama S, Narita S, Takahashi M, Sakurai T, Kawamura S, et al. The effect of treatment sequence on overall survival for men with metastatic castration-resistant prostate Cancer: a multicenter retrospective study. Clin Genitourin Cancer. 2020;18(2):e103-11. https://doi.org/10.1016/j. clgc.2019.09.006.

5. Okamoto T, Hatakeyama S, Narita S, Takahashi M, Sakurai T, Kawamura S, et al. Impact of nutritional status on the prognosis of patients with metastatic hormone-naïve prostate cancer: a multicenter retrospective cohort study in Japan. World J Urol. 2018;0123456789(9):1827-35. https:// doi.org/10.1007/s00345-018-2590-2.

6. Fizazi K, Tran N, Fein L, Matsubara N, Rodriguez-Antolin A, Alekseev BY, et al. Abiraterone plus prednisone in metastatic, castration-sensitive prostate Cancer. N Engl J Med. 2017;377(4):352-60. https://doi.org/10.1056/NEJMoa1 704174.

7. Suzuki H, Shin T, Fukasawa S, Hashine K, Kitani S, Ohtake N, et al. Efficacy and safety of abiraterone acetate plus prednisone in Japanese patients with newly diagnosed, metastatic hormone-naive prostate cancer: final subgroup analysis of LATITUDE, a randomized, double-blind, placebo-controlled, phase 3 study. Jpn J Clin Oncol. 2020;50(7):810-20. https://doi.org/10.1093/ jjco/hyaa030.

8. Mori K, Janisch F, Mostafaei H, Lysenko I, Karakiewicz PI, Enikeev DV, et al. Prognostic value of hemoglobin in metastatic hormone-sensitive prostate Cancer: a systematic review and meta-analysis. Clin Genitourin Cancer. 2020; 18(4):e402-9. https://doi.org/10.1016/j.clgc.2019.12.002. 
9. Sweeney CJ, Chen Y-H, Carducci M, Liu G, Jarrard DF, Eisenberger M, et al. Chemohormonal therapy in metastatic hormone-sensitive prostate Cancer. N Engl J Med. 2015;373(8):737-46. https://doi.org/10.1056/NEJMoa1503747.

10. Okamoto T, Hatakeyama S, Narita S, Arai Y, Habuchi T, Ohyama C. Validation and development of the CHAARTED criteria in patients with hormone-naïve metastatic prostate cancer: a multi-institutional retrospective study in Japan. Int J Urol. 2020;27(1):90-1. https://doi.org/10.1111/iju.14136.

11. Sato H, Narita S, Tsuchiya N, Koizumi A, Nara T, Kanda S, et al. Impact of early changes in serum biomarkers following androgen deprivation therapy on clinical outcomes in metastatic hormone-sensitive prostate cancer. BMC Urol. 2018;18(1):32. https://doi.org/10.1186/s12894-018-0353-4.

12. Nutritional anaemias. Report of a WHO scientific group. World Health Organ Tech Rep Ser. 1968;405:5-37 http://www.ncbi.nlm.nih.gov/pubmed/4975372. Accessed October 17, 2018.

13. Yamamoto $Y$, Hosogaya S, Osawa S, Ichihara K, Onuma T, Saito A, et al. Nationwide multicenter study aimed at the establishment of common reference intervals for standardized clinical laboratory tests in Japan. Clin Chem Lab Med. 2013;51(8):1663-72. https://doi.org/10.1515/cclm-2012-0413.

14. Soloway MS, Hardeman SW, Hickey D, Todd B, Soloway S, Raymond J, et al. Stratification of patients with metastatic prostate cancer based on extent of disease on initial bone scan. Cancer. 1988;61(1):195-202 http://www.ncbi. nlm.nih.gov/pubmed/3334948. Accessed August 9, 2018, DOl: 10.1002/10970142(19880101)61:1<195::AID-CNCR2820610133>3.0.CO;2-Y.

15. Scher HI, Halabi S, Tannock I, Morris M, Sternberg CN, Carducci MA, et al. Design and end points of clinical trials for patients with progressive prostate cancer and castrate levels of testosterone: recommendations of the prostate Cancer clinical trials working group. J Clin Oncol. 2008;26(7):114859. https://doi.org/10.1200/JCO.2007.12.4487.

16. Macciò A, Madeddu C, Gramignano G, et al. The role of inflammation, iron, and nutritional status in cancer-related anemia: results of a large, prospective, observational study. Haematologica. 2015;100(1):124-32. https://doi.org/10.3324/haematol.2014.112813.

17. Beer TM, Tangen CM, Bland LB, Thompson IM, Crawford ED. Prognostic value of anemia in newly diagnosed metastatic prostate cancer: a multivariate analysis of southwest oncology group study 8894. J Urol. 2004; 172(6 Pt 1):2213-7. http://www.ncbi.n/m.nih.gov/pubmed/15538234 Accessed October 17, 2018. https://doi.org/10.1097/01.ju.0000147771.92104. 83.

18. Nalesnik JG, Mysliwiec AG, Canby-Hagino E. Anemia in men with advanced prostate cancer: incidence, etiology, and treatment. Rev Urol. 2004;6(1):1-4 http://www.ncbi.nlm.nih.gov/pubmed/16985564. Accessed August 31, 2020.

19. Gupta D, Lis CG. Pretreatment serum albumin as a predictor of cancer survival: a systematic review of the epidemiological literature. Nutr J. 2010; 9(1):69. https://doi.org/10.1186/1475-2891-9-69.

20. Shamdas GJ, Ahmann FR, Matzner MB, Ritchie JM. Leukoerythroblastic anemia in metastatic prostate cancer. Clinical and prognostic significance in patients with hormone-refractory disease. Cancer. 1993;71(11):3594-600. https://doi.org/10.1002/1097-0142(19930601)71:11<3594:.:aid-cncr282 $0711121>3.0 . \mathrm{co} ; 2-\mathrm{O}$.

21. Vaupel $P$, Thews $O$, Hoeckel M. Treatment resistance of solid tumors. Med Oncol. 2001;18(4):243-60. https://doi.org/10.1385/MO:18:4:243.

22. Dunst J. Low hemoglobin levels: influence on tumor biology and radiotherapy treatment outcome. Eur J Cancer Suppl. 2004;2(2):3-10. https:// doi.org/10.1016/S1359-6349(03)00102-2.

23. Varlotto J, Stevenson MA. Anemia, tumor hypoxemia, and the cancer patient. Int J Radiat Oncol Biol Phys. 2005;63(1):25-36. https://doi.org/10.101 6/j.ijrobp.2005.04.049.

24. Semenza G. Signal transduction to hypoxia-inducible factor 1. Biochem Pharmacol. 2002;64(5-6):993-8. https://doi.org/10.1016/s0006-2952(02)011 68-1.

25. Fraga A, Ribeiro R, Príncipe P, Lopes C, Medeiros R. Hypoxia and prostate Cancer aggressiveness: a tale with many endings. Clin Genitourin Cancer. 2015;13(4):295-301. https://doi.org/10.1016/J.CLGC.2015.03.006.

26. Movsas B, Chapman JD, Hanlon AL, Horwitz EM, Greenberg RE, Stobbe C, et al. Hypoxic prostate/muscle po2 ratio predicts for biochemical failure in patients with prostate cancer: preliminary findings. Urology. 2002;60(4):6349. https://doi.org/10.1016/S0090-4295(02)01858-7.

27. Ragnum HB, Vlatkovic L, Lie AK, Axcrona K, Julin CH, Frikstad KM, et al. The tumour hypoxia marker pimonidazole reflects a transcriptional programme associated with aggressive prostate cancer. Br J Cancer. 2015;112(2):382-90. https://doi.org/10.1038/bjc.2014.604.
28. O'Reilly D, Johnson P, Buchanan PJ. Hypoxia induced cancer stem cell enrichment promotes resistance to androgen deprivation therapy in prostate cancer. Steroids. 2019;152(January):108497. https://doi.org/10.1016/j. steroids.2019.108497.

29. Fearon K, Arends J, Baracos V. Understanding the mechanisms and treatment options in cancer cachexia. Nat Rev Clin Oncol. 2013;10(2):90-9. https://doi.org/10.1038/nrclinonc.2012.209.

\section{Publisher's Note}

Springer Nature remains neutral with regard to jurisdictional claims in published maps and institutional affiliations.
Ready to submit your research? Choose BMC and benefit from:

- fast, convenient online submission

- thorough peer review by experienced researchers in your field

- rapid publication on acceptance

- support for research data, including large and complex data types

- gold Open Access which fosters wider collaboration and increased citations

- maximum visibility for your research: over $100 \mathrm{M}$ website views per year

At BMC, research is always in progress.

Learn more biomedcentral.com/submissions 